\title{
Comparing the Accuracy of Waist-Hip Ratio Calculation by the BIA device versus the Manual Method
}

\author{
${ }^{1}$ Amir Hossein Abedi Yekta, ${ }^{1}$ Shahrzad Khosravi ${ }^{*}$, ${ }^{1}$ Mohammad Hassabi, ${ }^{1}$ Mehrshad Poorsaid \\ Esfahani, ${ }^{1}$ Bahar Hassanmirzaei, ${ }^{1}$ Ahmadreza Asgari \\ ${ }^{1}$ Department of Sports Medicine, School of Medicine, Shahid Beheshti University of Medical Sciences, Tehran, Iran.
}

\begin{abstract}
Nowadays, the Waist-Hip Ratio is considered one of the most important indices in predicting the risk of cardiovascular diseases. The usual method for calculating this index is by measuring the waist and hip circumferences with a measuring tape. However, bioelectrical impedance analysis (BIA) devices are also capable of calculating this ratio. Hence, the goal of this study was to calculate this ratio with the BIA device and to compare it with the manual method done with measuring tape. A cross-sectional study was conducted on 270 individuals attending the Sports Medicine Department of Taleghani Hospital. Height, weight, waist and hip circumference were measured, and the body mass index (BMI) and waist-hip ratio (WHR) were calculated. Using the BIA technique, the body fat percentage (BFP) and WHR were calculated. The absolute and difference percentage between the values calculated by the device and measuring tape were calculated and the determinant factors of this difference were examined. The mean absolute difference between the two methods was 0.054 and the difference percentage was 0.064. Kappa's correlation coefficient between the two variables was 0.024, which indicates poor correlation between the findings of the two methods. Based on linear regression analysis, BFP, height, BMI, and total body water (TBW) significantly affected the difference percentage. On the contrary, age, waist circumference, hip circumference, and absolute body fat did not have such an effect. The absolute difference was 0.069 in women and 0.05 in men, which was statistically significant $(\mathrm{p}=0.025)$. Taking the manual waist-hip ratio method as the gold standard, the BIA method lacks adequate accuracy. Many variables such as, gender, BFP, TBW, BMI and height affect the accuracy of this method. Hence, until this technique is modified based on the aforementioned variables, we recommend avoiding the application of this method for WHR calculation.
\end{abstract}

KEY WORDS: Waist-Hip Ratio (WHR), Body Mass Index (BMI), Body Composition, Bioelectrical Impedance Analysis (BIA).

\section{INTRODUCTION}

The prevalence of obesity is rapidly on the rise in developing countries $(1,2)$. Inappropriate dietary regimes and lack of physical activity are among the most important factors contributing to obesity, which are themselves among the most important non-communicable diseases. Earlier studies have shown that both absolute body fat and distribution of central fat -which includes visceral fat- are closely associated with diseases such as diabetes, hypertension, raised fat levels and cardiovascular diseases (3). The morbidity

*. Corresponding Author:

Shahrzad Khosravi

E-mail: shahrzadekhosravi@yahoo.com 
and mortality associated with cardiovascular diseases in overweight men and women is three times that in other persons, such that $21 \%$ and $28 \%$ of these diseases in men and women, respectively, are attributed to obesity (4). Furthermore, the detection and screening of individuals before the presentation of cardiovascular diseases is very important. Although methods of visceral fat measurement, such as, computer tomography (CT) and magnetic resonance imaging (MRI) accurately illustrate the distribution of body fat (for the purpose of predicting metabolic diseases), but are very costly. Moreover, the risk of radiation does not allow these methods to be applied in epidemiologic research studies or to be employed by the individual. Thus, many different indices have been proposed for obesity (5). Body mass index (BMI) is one of these indices that is a reflection of total body fat, whereas, waist circumference (WC), waist-hip ratio (WHR), and waist-height ratio (WHtR) are indices applied for measuring central body fat (2). Based on literature, WC is the best and simplest index for measuring visceral fat tissue and may be the best index for predicting cardiovascular risk factors $(6,7)$. Nevertheless, considerable gender differences exist in the distribution of body fat. Thus, the appropriate anthropometric indices may differ in the two genders. Subsequently, the association between WHR \& BMI and the risk of ischemic heart disease was investigated in a study, which concluded that WHR might be a better predictor of ischemic heart disease (8). Elsewhere, body composition during weight loss in obese women was studied, wherein WHR was calculated by measuring WC and hip circumference (HC) with both measuring tape and the In body 2.0, Biospace device (South Korea). According to the results, both before and after dieting, the WHR calculated by the device was significantly greater than that measured by the measuring tape (9). Most studies that have employed anthropometric indices such as WC, HC and WHR have used a measuring tape[6]. Nevertheless, some studies have used the BIA device for this purpose (9). For example, one study in our country examined the BMI, WHR and BFP in'veterans hurt by chemical weapons' of Khorasan Razavi province with the Bioelectrical impedance analysis or BIA device, and concluded that these individuals required special planning and interventions as obesity is highly prevalent among them (10). Furthermore, the body composition of the employees of Azerbaijan's ShahidMadani University was evaluated with the BIA technique. Using the Zeus 9.9 device (South Korea) the WHR was calculated, where $33.9 \%$ of the women and $6.2 \%$ of the men fell in the high risk area (11).

In addition to the manual methods for WHR calculation recommended by the WHO (World Health Organization), nowadays other methods are applied too. One such method is the Bioelectrical Impedance Analysis (BIA) method. BIA is based on the principle that body tissues and components have different impedance properties against electrical current. For example, the electrical from that of water. Therefore, by placing the electrodes on different parts of the body (most often hands and feet), we may measure the current direction entered from one electrode to the other. The water in the body is localized in two compartments: extracellular water (ECW, approximately 45\%) and intracellular water (ICW, approximately 55\%) (12). On the other hand, the body also contains non-conducting materials (body fat) that provide resistance to the flow of electric current. Adipose tissue is significantly less conductive than muscle or bone. The principal of BIA is that electric current passes through the body at a differential rate depending on body composition. Based on the tissue water's electrical impedance, the device estimates the amount of body fat, muscle, bone and water; and calculates the WHR based on the data obtained (12).

Although different companies, so far, have made different devices, few studies have examined the accuracy of WHR calculated through manual measurement (with measuring tape) and that calculated by these devices. Therefore, in this study, we sought to examine the accuracy of WHR calculation by the BIA device as opposed to the manual method (by measuring tape).

\section{MATERIALS AND METHODS}

Participants and Measurement Protocol. A cross-sectional study was conducted on 270 individuals attending 'Taleghani Hospital's 
Sports Medicine Department to assess their health factors from $21^{\text {st }}$ Jan to $20^{\text {th }}$ March 2015. If the person cannot use the BIA, for example cause of Pregnancy, menstrual cycle or metal device in body, the case was exclude the study. After explaining the study to them and attaining written consent their age and genders were registered. Thereafter, their heights and weights were measured. Their weight was measured with a digital SECA scale (with an accuracy of $0.1 \mathrm{~kg}$ ), with the least clothing and without shoes. Height was measured with a measuring tape (with an accuracy of $0.1 \mathrm{~cm}$ ), without shoes, in standing position and arms held naturally. BMI was calculated by dividing weight $(\mathrm{kg})$ by square meter of height $(\mathrm{m})$. Based on WHO's recommendation, WC should be measured approximately midpoint between the lower margin of the last palpable rib and the top of the iliac crest (13). The United States (US) National Institutes of Health however, recommends measuring $\mathrm{WC}$ at the top of the iliac crest (14). Although there are different protocols for WC measurement, all of them unanimously recommend measuring $\mathrm{HC}$ at the widest part of the buttocks. The accuracy of the WC and HC measurements depend on the tightness of the measuring tape. According to the $W H O$, the measuring tape should go round the body and be held parallel to the floor; however, it should not be held too tightly (1). Moreover, the use of a stretch-resistant tape that creates a constant pressure of $100 \mathrm{~g}$ has been advised to minimize the differences in measurement tightness. Therefore, we measured the WC at the slimmest part of the waist and measured the $\mathrm{HC}$ at the most prominent part of the buttocks, with the least clothing, and with a measuring tape (with an accuracy of $0.1 \mathrm{~cm}$ ). The WHR was calculated by dividing the WC by the HC. The body fat percentage (BFP) was measured through the BIA technique and with the GAIA Plus device (Jawon Medical, South Korea). To measure BFP, the resistance of the entire body against $50 \mathrm{KHz}$ was measured after 5 hours of nocturnal fasting, an emptied bladder and having put away any jewelry or metallic substances. The age, gender and height of the participant were entered into the device and the device calculated the weight itself.
Statistical Analysis. Statistical analysis was performed with SPSS 22 software. We described the qualitative variables using frequency and frequency percent, and quantitative variables with mean and standard deviation. To obtain the difference between the WHR calculated by the two methods, the WHR calculated through measuring tape was deducted from the WHR calculated with the BIA. The difference was calculated in absolute value and the difference percentage was attained by dividing the difference by the numbers calculated manually. T-test was used to compare the difference from zero. Independent t-test was used to examine the difference in values measured in the two genders. Pearson's correlation coefficient was used to evaluate the correlation between the values measured. $\mathrm{P}<0.05$ was considered as the level of significance.

Ethical considerations. The Ethics Committee of Taleghani Hospital approved the study. We took written consent from all the patients. They were reassured that their information would remain confidential and that it would only be shared with their physician if need be.

\section{RESULTS}

Among the 270 participants of the study, 57 $(21 \%)$ were females and $213(79 \%)$ were males. The mean and range of age of the participants were $39.9 \pm 8.8$ and $24-66$ years, respectively.

Table 1 shows the range and mean of the quantitative data, including, height, weight, BMI, waist circumference, hip circumference, waist-hip ratio calculated by both methods, absolute value of body fat, body fat percentage, and total body water (TBW).

Both the means of WHR calculated with BIA and manually were 0.88 , which can be misleading. The purpose of this research was to examine the mean difference between the two values calculated by both methods. To this end, we obtained the absolute difference (per person) by subtracting the values from each other. Moreover, the difference percentage for each person was calculated by dividing the absolute difference by the value derived manually. The mean absolute 
difference between the two methods was 0.054 , and the difference percentage was 0.064 .

The Kappa measure of agreement between the WHR calculated by the two methods was 0.024 , which indicates a poor correlation between the two methods' findings. Based on the results of linear regression analysis, BFP, height, BMI, and TBW significantly affected the difference percentage; while, age, WC, $\mathrm{HC}$, and absolute body fat did not have such an effect (Table 3).

Table 1. Descriptive quantitative data.

\begin{tabular}{lccccc}
\hline Variable & Number & Minimum & Maximum & Mean & Standard deviation \\
\hline Age & 270 & 24.00 & 66.00 & 39.9296 & 8.82158 \\
\hline Height & 270 & 147.00 & 200.00 & 172.8815 & 8.26978 \\
\hline Weight & 270 & 48.10 & 143.90 & 78.4567 & 14.63140 \\
\hline BMI & 270 & 18.20 & 41.50 & 26.1111 & 3.65112 \\
\hline WC with measuring tape & 270 & 60.00 & 126.00 & 89.2037 & 11.85190 \\
\hline HC with measuring tape & 270 & 81.00 & 132.00 & 100.8037 & 6.65915 \\
\hline WHR calculated manually & 270 & 0.65 & 1.09 & 0.8834 & 0.08916 \\
\hline WHR calculated with BIA & 270 & 0.66 & 1.05 & 0.8854 & 0.07061 \\
\hline Body fat (kg) & 270 & 3.90 & 48.90 & 19.5678 & 6.30683 \\
\hline BFP & 270 & 7.80 & 38.00 & 24.7233 & 5.51545 \\
\hline TBW & 270 & 23.80 & 68.40 & 42.3985 & 7.67597 \\
\hline
\end{tabular}

Table 2. The statistical analysis of quantitative variables for each gender, separately.

\begin{tabular}{|c|c|c|c|c|c|}
\hline Variable & & Number & Mean & $\begin{array}{l}\text { Standard } \\
\text { deviation }\end{array}$ & $\begin{array}{c}\mathbf{P} \\
\text { value }\end{array}$ \\
\hline \multirow{2}{*}{ BMI } & Female & 57 & 24.1246 & 3.21866 & \multirow{2}{*}{$0.001^{* *}$} \\
\hline & Male & 213 & 26.6427 & 3.58236 & \\
\hline \multirow{2}{*}{ Body fat } & Female & 57 & 18.5860 & 5.24723 & \multirow{2}{*}{0.186} \\
\hline & Male & 213 & 19.8305 & 6.54745 & \\
\hline \multirow{2}{*}{ BFP } & Female & 57 & 28.4053 & 5.13790 & \multirow{2}{*}{$0.001^{* *}$} \\
\hline & Male & 213 & 23.7380 & 5.19671 & \\
\hline \multirow{2}{*}{ WC } & Female & 57 & 75.0351 & 6.25061 & \multirow{2}{*}{$0.001^{* * *}$} \\
\hline & Male & 213 & 92.9953 & 9.97473 & \\
\hline \multirow{2}{*}{ HC } & Female & 57 & 99.6842 & 6.40635 & \multirow{2}{*}{0.153} \\
\hline & Male & 213 & 101.1033 & 6.70811 & \\
\hline \multirow{2}{*}{ WHR calculated manually } & Female & 57 & .7535 & .04809 & \multirow{2}{*}{$0.001^{* * *}$} \\
\hline & Male & 213 & .9182 & .06100 & \\
\hline \multirow{2}{*}{ WHR calculated with BIA } & Female & 57 & .8135 & .05749 & \multirow{2}{*}{$0.001^{* *}$} \\
\hline & Male & 213 & .9046 & .06077 & \\
\hline \multirow{2}{*}{ TBW } & Female & 57 & 33.4246 & 6.06831 & \multirow{2}{*}{$0.001^{* * *}$} \\
\hline & Male & 213 & 44.8000 & 6.13094 & \\
\hline \multirow{2}{*}{ WHR difference between the two methods } & Female & 57 & .0698 & .06010 & \multirow{2}{*}{$0.025^{*}$} \\
\hline & Male & 213 & .0504 & .04373 & \\
\hline \multirow{2}{*}{$\begin{array}{l}\text { WHR difference percentage between the two } \\
\text { methods }\end{array}$} & Female & 57 & .0957 & .08550 & \multirow{2}{*}{$0.001^{* *}$} \\
\hline & Male & 213 & .0556 & .05077 & \\
\hline
\end{tabular}

**: significant at $\mathrm{p}<0.01$.

*: significant at $\mathrm{p}<0.05$.

Abedi, A. H. Yekta, et al. (2016). Ann Appl Sport Sci, 4(2): 09-15. 
Table 3. Coefficients between WHR ,Body fat and total Body water

\begin{tabular}{cccccc}
\hline \multirow{2}{*}{ Model } & \multicolumn{2}{c}{$\begin{array}{c}\text { Unstandardized } \\
\text { Coefficients }\end{array}$} & Standardized Coefficients & \multirow{2}{*}{ t } & Sig. \\
\cline { 2 - 5 } & B & Std. Error & Beta & -1.404 & .162 \\
\hline (Constant) & -.812 & .579 & -.025 & -.422 & .673 \\
\hline Age & .000 & .000 & 1.273 & 3.096 & $.002^{* *}$ \\
\hline Height & .010 & .003 & 2.162 & 3.586 & $.000^{* *}$ \\
\hline BMI & .037 & .010 & -.313 & -.540 & .590 \\
\hline Hip Circumference & -.003 & .005 & 1.015 & .875 & $.382^{* *}$ \\
\hline Waist Circumference & .005 & .006 & -.882 & -2.855 & $.005^{* *}$ \\
\hline Body fat percent & -.010 & .003 & -.436 & -1.046 & .297 \\
\hline Body fat & -.004 & .004 & -2.294 & -3.488 & $.001^{* *}$ \\
\hline Total body water & -.018 & .005 & & & \\
\hline
\end{tabular}

a. Dependent Variable: diffpercent2

**: significant at $\mathrm{p}<0.01$.

The absolute differences in females and males were 0.069 and 0.05 , respectively, which were statistically significant ( $\mathrm{p}$ value $=0.025)$.

Using independent sample t-test, this difference was assessed in different groups of different fat percentages. The difference percentage in individuals with BFP between 20 and 30 was 0.55 ; and was 0.08 in persons with BFP of lower or higher values. This difference was statistically significant ( $p$ value $=0.004$ ).

Furthermore, the difference percentage in people with BMI between 25 to 30 was 0.055 , and was 0.07 in others with different BMIs. This difference too, was statistically significant ( $p$ value $=0.026$ ).

\section{DISCUSSION}

Based on our findings, the waist-hip ratio calculated by the BIA technique significantly differs from that measured by measuring tape. A 0.024 kappa measure of agreement between the WHR calculated by the two methods indicates a poor agreement between the two. If we consider the manual method as the gold standard, we may conclude that the WHR calculated by the BIA device is poorly associated with reality.

Therefore, we recommend using the measuring tape for the estimation of WHR. Alternatively, the ratios calculated by the BIA device should be adapted to the ratios obtained manually, and the cut-off points should be modified.
Here, we observed that women differ from men in their anthropometric indices, including, BMI, BFP, WC, WHR calculated by both methods, and TBW, which is in line with the findings of earlier studies $(2,6,7)$. This very issue may be the main reason behind the significant variation in difference percentage between the two methods in men and women.

In addition to gender, many other factors influence the difference between the two methods. These are, BFP, height, BMI and TBW (15)

The findings of previous study indicate that BIA significantly overestimated $\% \mathrm{BF}$ in men and women by less than $1 \%$ (16). BIA is a valid method to estimate \%BF in adults with BMI classified as Normal or Overweight person, but not as Obese adult. Estimation of trunk resistance with current BIA devices may explain the underestimation of $\% \mathrm{BF}$ in the adults with obese person (16). We observed that people with a 25 - 30 BMI had the least difference compared to people with lower or higher BMIs. Furthermore, fat percentage was another factor shown to have affected this difference. The WHR calculated by BIA in individuals with 20 to 30 fat percentages, too, exhibited the least difference with the standard level compared to individuals with different BFPs.

Menopause changes fat distribution and women experience a loss in lean mass and an increase in weight, fat mass and central fat deposition (17-21). The ratio of fat/lean mass, 
especially in the lower part of the body increases $(22,23)$, which may affect the estimated impedance as the current passes through the legs.

\section{CONCLUSION}

To examine and compare the difference between WHR calculation by methods other than the manual method (with measuring tape), we recommend conducting studies on bigger sample sizes and the employment of other devices. If need be, the cut-off points should also be standardized based on the method of measurement. If the manual method of WHR calculation is considered the gold standard, based on our results, measuring this index through the BIA device lacks the required accuracy. Since many variables such as, BFP, TBW, BMI and height affect the accuracy of this technique, we would recommend not using this technique for WHR estimation, unless and until it has been modified on the basis of the aforementioned variables.

\section{APPLICABLE REMARKS}

- BIA method analysis is very simple and quick to access WHR, but the result must be modified on the variable.

\section{ACKNOWLEDGMENTS}

We are deeply grateful to the staff at Taleghani Hospital's Sports Medicine Department for providing us with the data used in this research.

\section{REFERENCES}

1. Obesity: preventing and managing the global epidemic. Report of a WHO consultation. World Health Organization technical report series. 2000;894:i-xii, 1-253.

2. Kamadjeu RM, Edwards R, Atanga JS, Kiawi EC, Unwin N, Mbanya JC. Anthropometry measures and prevalence of obesity in the urban adult population of Cameroon: an update from the Cameroon Burden of Diabetes Baseline Survey. BMC public health. 2006;6:228.

3. Esmaillzadeh A, Mirmiran P, Azizi F. Comparative evaluation of anthropometric measures to predict cardiovascular risk factors in Tehranian adult women. Public health nutrition. 2006;9(1):61-9.

4. Esmaillzadeh A, Mirmiran P, Azizi F. Waist-to-hip ratio is a better screening measure for cardiovascular risk factors than other anthropometric indicators in Tehranian adult men. International journal of obesity and related metabolic disorders : journal of the International Association for the Study of Obesity. 2004;28(10):1325-32.

5. Yang CY, Peng CY, Liu YC, Chen WZ, Chiou WK. Surface anthropometric indices in obesity-related metabolic diseases and cancers. Chang Gung medical journal. 2011;34(1):1-22.

6. Dobbelsteyn CJ, Joffres MR, MacLean DR, Flowerdew G. A comparative evaluation of waist circumference, waist-to-hip ratio and body mass index as indicators of cardiovascular risk factors. The Canadian Heart Health Surveys. International journal of obesity and related metabolic disorders : journal of the International Association for the Study of Obesity. 2001;25(5):652-61.

7. Olinto MT, Nacul LC, Gigante DP, Costa JS, Menezes AM, Macedo S. Waist circumference as a determinant of hypertension and diabetes in Brazilian women: a population-based study. Public health nutrition. 2004;7(5):62935 .

8. Yusuf S, Hawken S, Ounpuu S, Bautista L, Franzosi MG, Commerford P, et al. Obesity and the risk of myocardial infarction in 27,000 participants from 52 countries: a case-control study. Lancet. 2005;366(9497):1640-9.

9. Kim HJ, Gallagher D, Song MY. Comparison of body composition methods during weight loss in obese women using herbal formula. The American journal of Chinese medicine. 2005;33(6):851-8.

10. Hashemi Javaheri SAA, Saberi M, Mohamad Rahimi N, Khodaei M. Body Mass Index, Waist to Hip Ratio, and Percentage of Body Fat of the Chemical War Survivors in Razavi Khorasan. Tebe-E-Janbaz. 2012;4(14):[Article in Farsi].

11. Ghorbanian B. The Assessment of Body Composition by Bioelectrical Impedance Analysis among Personals of Iranian Azerbaijan Shahid Madani University. Sport Physiology (Research on Sport Science). 2013;5(17):115-29 [Article in Farsi].

12. Kyle UG, Bosaeus I, De Lorenzo AD, Deurenberg P, Elia M, Manuel Gomez J, et al. Bioelectrical impedance analysis-part II: utilization in clinical practice. Clinical nutrition. 2004;23(6):1430-53. 
13. World Health Organization. 2008-2013 Action Plan for the Global Strategy for the Prevention and Control of Noncommunicable Diseases. In: Organization WH, editor. 2008.

14. Centers for Disease Control and Prevention. National Health and Nutrition Examination Survey. 2011.

15. Dehghan M, Merchant AT. Is bioelectrical impedance accurate for use in large epidemiological studies? Nutrition journal. 2008;7:26.

16. Shafer KJ, Siders WA, Johnson LK, Lukaski HC. Validity of segmental multiple-frequency bioelectrical impedance analysis to estimate body composition of adults across a range of body mass indexes. Nutrition. 2009;25(1):25-32.

17. Macdonald HM, New SA, Campbell MK, Reid DM. Longitudinal changes in weight in perimenopausal and early postmenopausal women: effects of dietary energy intake, energy expenditure, dietary calcium intake and hormone replacement therapy. International journal of obesity and related metabolic disorders : journal of the International Association for the Study of Obesity. 2003;27(6):669-76.

18. Panotopoulos G, Raison J, Ruiz JC, Guy-Grand B, Basdevant A. Weight gain at the time of menopause. Human reproduction. 1997;12 Suppl 1:126-33.

19. Toth MJ, Gardner AW, Ades PA, Poehlman ET. Contribution of body composition and physical activity to agerelated decline in peak VO2 in men and women. Journal of applied physiology (Bethesda, Md : 1985). 1994;77(2):647-52.

20. Wang Q, Hassager C, Ravn P, Wang S, Christiansen C. Total and regional body-composition changes in early postmenopausal women: age-related or menopause-related? The American journal of clinical nutrition. 1994;60(6):843-8.

21. Zamboni M, Turcato E, Santana H, Maggi S, Harris TB, Pietrobelli A, et al. The relationship between body composition and physical performance in older women. Journal of the American Geriatrics Society. 1999;47(12):1403-8.

22. Malina RM, Huang YC, Brown KH. Subcutaneous adipose tissue distribution in adolescent girls of four ethnic groups. International journal of obesity and related metabolic disorders : journal of the International Association for the Study of Obesity. 1995;19(11):793-7.

23. Reubinoff BE, Wurtman J, Rojansky N, Adler D, Stein P, Schenker JG, et al. Effects of hormone replacement therapy on weight, body composition, fat distribution, and food intake in early postmenopausal women: a prospective study. Fertility and sterility. 1995;64(5):963-8. 